\title{
Empire Europa: Das militärische Fundament der Wirtschaftsmacht EU
}

Die Europäische Union ist derzeit dabei, sich grundlegend zu verändern. Beleg hierfür sind etwa die Aussagen von EU-Erweiterungskommissar Olli Rehn, der die Union als ein »gutmütiges Imperium $^{\mathrm{I}}$ bezeichnet, oder von Kommissionschef José Manuel Barroso, der in ihr ebenfalls »eine Art Imperium « sieht. ${ }^{2}$ Die Zeit falscher Bescheidenheit ist vorbei, offensiv formulieren die Verantwortlichen in der EU und den Mitgliedstaaten inzwischen den Anspruch, in den Kreis der Großmächte aufsteigen zu wollen. Die im Jahr 2000 veröffentlichte Lissabon-Strategie mit ihrer Zielvorgabe, innerhalb von zehn Jahren zur Weltwirtschaftsmacht Nummer eins aufzusteigen, stellt in diesem Zusammenhang die wirtschaftliche Komponente der »Supermacht EU« dar. Erreicht werden soll dieses ehrgeizige Ziel einerseits durch einen neoliberalen Umbau innerhalb der Mitgliedstaaten - in Deutschland etwa mit der Agenda 2010 und dem damit einhergehenden massiven Sozialabbau - sowie durch die Durchsetzung der eigenen Liberalisierungsagenda nach außen.

Nicht von ungefähr wurde nahezu parallel mit einem umfassenden Programm zur Militarisierung der Europäischen Union begonnen, um dem ökonomischen Weltmachtanspruch ein militärisches Fundament zu verschaffen. Dieser Beitrag zeichnet die wichtigsten Stationen, Dokumente und Komponenten dieser Entwicklung nach und analysiert sie im Kontext einer Europäischen Sicherheitsund Verteidigungspolitik (ESVP), die immer offener auf die Durchsetzung eigener Machtansprüche abzielt. Im Kern geht es dabei inhaltlich um drei Dinge: Einfluss im Weltmaßstab - auch gegen die USA, Rohstoffkontrolle, und die militärische Absicherung der neoliberalen Weltwirtschaftsordnung.

\section{Stationen und Komponenten der Militarisierung Europas}

Obwohl die Gemeinsame Außen- und Sicherheitspolitik (GASP) bereits Anfang der I 990er mit dem Vertrag von Maastricht als eine der drei zentralen Säulen der Europäischen Union eingeführt wurde, führte sie lange Jahre eher ein Schattendasein. Erst das französisch-britische Treffen in St. Malo Ende I998 ebnete grundsätzlich den Weg für eine Militarisierung der Europäischen Union. Schon im Juni des darauf folgenden Jahres beschlossen die Staats- und Regierungschefs, eine EU-Eingreiftruppe aufzustellen. Mit der Verabschiedung des »European Headline Goal« durch Beschluss des Europäischen Rates nach Art. I 3 Abs. I i.V.m. Art. 23 Abs. I EUV im Dezember $1999^{3}$ wurde der Umfang dieser Truppe auf 60.000 Soldaten festgelegt, von denen Deutschland ca. ein Drittel stellt. Berücksichtigt man die für ein solches Kontingent notwendige Rotation, müssen je nach Schätzung I 50.000 bis I 80.000 Soldaten vorgehalten werden. Interessant ist der Aktionsradius, der inzwischen für einsatzbereit erklärten Truppe. Er wurde zunächst auf 4.000 Kilometer rund um Brüssel festgelegt, was in seiner weltumspannenden Reichweite bereits indiziert, dass es hier um die Etablierung

\footnotetext{
I Alan Posener, Globale Politik - Warum Europa das Zeug zur Weltmacht hat, Die Welt vom I 5.9.2007, 7 .

2 »Dimensionen eines Imperiums (Interview mit José Manuel Barroso), Die Welt vom 17.10.2007, 3.

3 Europäischer Rat Helsinki Io./I I. I 2.1999 Schlussfolgerung des Vorsitzes Anlage IV.
} 
einer global agierenden Interventionsarmee zur Durchsetzung europäischer Interessen geht.

Auf der Tagung des Europäischen Rates im Dezember 2000 in Nizza wurden auf der Grundlage der Art. I I ff. EUV mit dem Beschluss zur Einsetzung eines Militärausschusses (EUMC), eines Militärstabes (EUMS) und eines ständigen Politischen und Sicherheitspolitischen Komitees (PSK) auch die organisatorischen Rahmenbedingungen für offensiv ausgerichtete EU-Truppen geschaffen. Derart aufgestellt, wurde das neue militärische Selbstverständnis rasch Praxis: Die ersten ESVP-Einsätze, »Concordia « in Mazedonien und »Artemis « im Kongo, fanden bereits im Jahr 2003 statt. Letzterer ist aus zwei Gründen interessant: Einmal liegt das Einsatzgebiet deutlich weiter als 4.000 Kilometer von Brüssel entfernt, womit auch diese räumlich ohnehin schon sehr weit gefasste Einschränkung endgültig ad acta gelegt wurde. Zudem agierte die EU im Kongo erstmalig ohne Rückgriff auf NATO-Strukturen und damit unabhängig von den USA, indem Frankreich die operative Führung übernahm. Im Dezember 2004 übernahm die Europäische Union die NATO-Mission in Bosnien-Herzegowina. Dieser »Althea«genannte Einsatz umfasst bis zu 7.000 Soldaten, bereits diese Zahl manifestiert die zunehmende Bedeutung der EU-Streitkräfte. Seither kommen immer häufiger weitere Einsätze hinzu, mittlerweile fanden über 20 Missionen im Rahmen der Europäischen Sicherheits- und Verteidigungspolitik statt. 4

Im Juni 2004 wurde darüber hinaus eine neue militärische Zielvorgabe, das »Headline Goal 2010«, vom Europäischen Rat beschlossen, die u.a. den Aufbau von EU-Kampftruppen (sog. Battlegroups) vorsieht. Diese I.500 Soldaten umfassenden, hochflexiblen Kampfeinheiten sollen innerhalb von 5 bis 30 Tagen vor Ort stationiert werden können. Seit Januar 2007 stehen die ersten der insgesamt 22 bislang geplanten Kampfeinheiten zur Verfügung, die im Übrigen auch explizit ohne UN-Mandat eingesetzt werden können.'

Ebenfalls seit 2007 verfügt die EU auch über eine so genannte Zivil-militärische Zelle zur Planung und Durchführung von Einsätzen im Umfang von bis zu 2.000 Soldaten (zuvor war man entweder auf nationale Kapazitäten oder auf die NATO und damit auf die USA angewiesen). Sie bildet den Nukleus für ein voll funktionsfähiges Hauptquartier, mit dem sich die Europäische Union künftig weiter von den USA emanzipieren, also unabhängiger machen will.

\section{Globalmacht Europa}

Einen »Meilenstein « (so Fraser Cameron, ehemaliger Berater der Europäischen Kommission und derzeit Direktor des EU-Russia Centre in Brüssel) für die Fortentwicklung der europäischen Militärkomponente stellte die im Dezember 2003 verabschiedete Europäische Sicherheitsstrategie (ESS) dar. Mit ihr erhebt die Europäische Union unmissverständlich den Anspruch, im Konzert der Supermächte eine führende Rolle zu spielen: »Als Zusammenschluss von 25 Staaten mit über 450 Millionen Einwohnern, die ein Viertel des Bruttosozialprodukts (BSP) weltweit erwirtschaften, ist die Europäische Union [...] zwangsläufig ein globaler Akteur. [...] Europa muss daher bereit sein, Verantwortung für die globale Sicherheit und für eine bessere Welt mit zu tragen. ${ }^{6}$

\footnotetext{
4 Allein im Jahr 2008 wurden bis Mai drei Einsätze (im Tschad, in Guinea-Bissau und im Kosovo) begonnen.

5 Otfried Nassauer, Europas schnelle Eingreifverbände, Berlin-Information Center for Transnational Security-Stichwort, November 2004.

6 Europäische Sicherheitsstrategie, Ein sicheres Europa in einer besseren Welt, Brüssel I 2.I 2.2003.
} 
Gleichzeitig identifiziert die EU-Sicherheitsstrategie eine Reihe von Bedrohungen, gegen die frühzeitig militärisch vorgegangen werden müsse: »Die erste Verteidigungslinie wird oftmals im Ausland liegen. [...] Wir müssen eine Strategiekultur entwickeln, die ein frühzeitiges, rasches und wenn nötig robustes Eingreifen fördert. «7 Damit übernimmt die ESS indirekt die US-amerikanische Präventivkriegsstrategie, wobei sie als eine wesentliche Bedrohung u.a. die zunehmende Abhängigkeit von Rohstoffimporten benennt.

Schon dieser kurze Überblick zeigt, wie weit die Militarisierung Europas bereits fortgeschritten ist. Mit dem Lissabonner Vertrag soll sie jedoch entscheidend weiter vorangetrieben werden.

\section{Militarisierung per Vertrag}

Ursprünglich, d.h. vor der Ablehnung im irischen Referendum, sollte der »Lissabonner Vertrag « bis spätestens Sommer 2009 als neue Rechtsgrundlage der Union in Kraft treten. ${ }^{8}$ Obwohl bereits sein Vorgänger, der EU-Verfassungsvertrag, bei Referenden in Frankreich und den Niederlanden im Jahr 2005 abgelehnt wurde, gelang es den EU-Regierungen, alle wesentlichen Punkte in den Vertrag von Lissabon hinüberzuretten, wie eine Presseerklärung der Bundesregierung unumwunden einräumt: »Der Begriff >Verfassung für Europa< war nach der Ablehnung bei den Volksabstimmungen in Frankreich und den Niederlanden nicht mehr haltbar. Das erklärte Ziel der deutschen Ratspräsidentschaft war es aber, die Substanz der Verfassung zu erhalten. Dies konnte erreicht werden.«9 Diese Substanz besteht neben dem schon in den Gründungsverträgen festgeschriebenen neoliberalen Wirtschaftsmodell insbesondere in den neuen Regelungen im Militärbereich. So wird mit dem Lissabonner Vertrag das mögliche Aufgabenspektrum für EU-Kampfeinsätze substanziell erweitert. In Art. 43 (I) werden hierfür u.a. »die Unterstützung für Drittländer bei der Bekämpfung des Terrorismus in ihrem Hoheitsgebiet «, »Kampfeinsätze im Rahmen der Krisenbewältigung «, »Operationen zur Stabilisierung der Lage«, also Besatzungsmissionen wie in Afghanistan und sogar "gemeinsame Abrüstungsmaßnahmen« benannt, eine Formulierung, die verdächtig an die US-amerikanische Begründung für den Angriffskrieg gegen den Irak erinnert. Extrem weit reichend ist auch die so genannte "Solidaritätsklausel « in Art. 222 (гa), mit dem der Lissabonner Vertrag festschreibt, dass die EU »alle ihr zur Verfügung stehenden Mittel, einschließlich der ihr von den Mitgliedstaaten bereitgestellten militärischen Mittel« mobilisiert, um »terroristische Bedrohungen im Hoheitsgebiet von Mitgliedstaaten abzuwenden." Mit diesem Artikel wird die Europäische Union nicht nur zu einem Militärbündnis, er eröffnet zudem auch noch die Option zum Einsatz von EU-Militär im Inneren. Dies ist überaus problematisch, da die EUJustizminister bereits im Dezember 200 I eine Rahmenerklärung verabschiedeten, in der es heißt, Terrorismus beinhalte auch Aktivitäten, die in der Absicht erfolgen, »öffentliche Körperschaften oder eine internationale Organisation unangemessenem Zwang auszusetzen, damit sie bestimmte Handlungen unter-

\footnotetext{
7 Ebd., $6 \mathrm{ff}$.

8 Rat der Europäischen Union, Konsolidierte Fassungen des Vertrags über die Europäische Union und des Vertrags über die Arbeitsweise der Europäischen Union, Brüssel, den I 5.4.2008. Die im Folgenden angegebenen Artikelnummern beziehen sich auf diese Fassung des Vertrages.

9 Staats- und Regierungschefs verabschieden Reform der Europäischen Union, e-public, das Europa-Magazin, Nr. $50 / 2007$.
} 
nehmen oder unterlassen. «을 Interessant ist in diesem Kontext, dass die viel gerühmte Grundrechtecharta offensichtlich die gezielte Tötung zur Aufstandsbekämpfung legitimiert: »Eine Tötung wird nicht als Verletzung dieses Artikels [Art. 2. Abs. I: Recht auf Leben, T.P.] betrachtet, wenn sie durch eine Gewaltanwendung verursacht wird, die unbedingt erforderlich ist, um [...] einen Aufruhr oder Aufstand rechtmäßig niederzuschlagen. «II

Des Weiteren wird der Lissabonner Vertrag einen regelrechten Rüstungsschub ermöglichen. Denn mit Art. 4I wird erstmals die Möglichkeit zur Aufstellung eines EU-Rüstungshaushalts, euphemistisch »Anschubfonds « genannt, eröffnet, was bislang durch den noch gültigen Vertrag von Nizza untersagt ist. Darüber hinaus schreibt Art. 42 (3) den Mitgliedsstaaten in bislang einzigartiger Weise vor, militärisch aufzurüsten: »Die Mitgliedstaaten verpflichten sich, ihre militärischen Fähigkeiten schrittweise zu verbessern.«Um die Einhaltung dieser Verpflichtung zu kontrollieren, wurde bereits im Juni 2004 die »Europäische Verteidigungsagentur« ins Leben gerufen. Dass dieses Organ in einem frühen Entwurf der EU-Verfassung noch explizit als »Europäisches Amt für Rüstung, Forschung und militärische Fähigkeiten « bezeichnet wurde, macht ihre eigentliche Zielsetzung ebenso deutlich, wie frühe Versuche, in die Aufrüstungsverpflichtung eine bindende Zielgröße von $2 \%$ des Bruttoinlandsprodukts für Rüstungsausgaben aufzunehmen. Dies hätte für Deutschland eine Steigerung um etwa $30 \%$ zur Folge gehabt.

Besonders besorgniserregend ist, dass hinsichtlich der Entsendung von EUKampftruppen die Gewaltenteilung auf EU-Ebene faktisch außer Kraft gesetzt ist. So spricht Art. 275 dem Europäischen Gerichtshof keinerlei Kontrollbefugnisse bzgl. der EU-Militärpolitik zu. Auch das Europäische Parlament ist nicht zuständig, es wird gemäß Art. 36 lediglich regelmäßig darüber »unterrichtet«, was die Exekutive in diesem Bereich unternimmt. Das Parlament darf zwar »Anfragen oder Empfehlungen an den Rat und den Hohen Vertreter richten «, zu entscheiden hat es aber nichts. Ohnehin stellt sich die Frage, wie die kurze Einsatzzeit der Battle Groups ( 5 bis 30 Tage) mit dem deutschen Parlamentsvorbehalt $^{12} \mathrm{zu}$ vereinbaren ist. Die damalige britische Ratspräsidentschaft antwortete dem Autor auf diese Frage im Unterausschuss Sicherheit und Verteidigung des Europäischen Parlaments, die deutschen Kollegen hätten angemerkt, dass eine Zustimmung des Bundestages unter Umständen auch im Nachhinein möglich sei. In dieses Bild passt, dass der parlamentarische Staatssekretär im Verteidigungsministerium Christian Schmidt (CSU) Vorratsbeschlüsse für EU Battle Groups und NATO Response Force fordert. ${ }^{13}$ Genau dies hat die im Mai 2008 veröffentlichte »Sicherheitsstrategie für Deutschland « der CDU/CSU-Bundestagsfraktion nun auch offiziell vorgeschlagen. ${ }^{14}$ Diese drastische Einschränkung demokratischer Kontrollmöglichkeiten bei einer solch entscheidenden Frage ist nicht hinnehmbar, da sie zur Folge hat, dass die Exekutive das EU-Militär

Io Jean-Claude Paye, Ausnahmezustand in Permanenz, Blätter für deutsche und internationale Politik 9/ 2006, 1089-1096, 1093.

I I Erläuterungen zur Charta der Grundrechte, 2007/C 303/02, I f., wo auf Art. 2 Abs. 2 lit. C EMRK Bezug genommen wird.

I 2 BVerfGE 90, 286 (38 I ff.); Gesetz über die parlamentarische Beteiligung bei der Entscheidung über den Einsatz bewaffneter Streitkräfte im Ausland (Parlamentsbeteiligungsgesetz), BGBl. 2005 I, $775 \mathrm{ff}$.

I 3 Handelsblatt vom 7.I.2007: CSU-Staatssekretär will »Doppelbeschluss « für Auslandseinsätze.

I4 Eine Sicherheitsstrategie für Deutschland, Beschluss der CDU/CSU-Bundestagsfraktion vom 6.5.2008. 
nahezu beliebig einsetzen kann. Nachdem bereits das Europäische Parlament über keinerlei Mitentscheidungsrechte hinsichtlich der Frage von EU-Militäreinsätzen verfügt, soll mit der »Sicherheitsstrategie für Deutschland « nun auch die Hoheit des Deutschen Bundestages ausgehebelt und somit die formale Gewaltenteilung in der entscheidenden Frage von Krieg und Frieden de facto aufgehoben werden.

\section{Kerneuropakonzept konkret}

Neben der forcierten Militarisierung soll der Lissabonner Vertrag vor allem eine massive Machtverschiebung zugunsten der mächtigen, bevölkerungsreichen EU-Länder bewerkstelligen. Hierfür sind zwei Elemente entscheidend. Einmal ermöglicht Art. 46 die Einführung einer »Ständigen Strukturierten Zusammenarbeit «, mit der einzelne Mitgliedstaaten ganz im Sinne des Kerneuropa-Prinzips Exklusivgruppen im militärischen Bereich bilden können. Da sich dabei das Einstimmigkeitsprinzip »allein auf die Stimmen der Vertreter der an der Zusammenarbeit teilnehmenden Mitgliedstaaten « bezieht, wird hierdurch die bislang geltende Konsenspflicht im Militärbereich ausgehebelt. Zudem regelt Protokoll ı०, dass nur die Mitgliedsländer an der »Ständigen Strukturierten Zusammenarbeit« teilnehmen dürfen, die sich an den wichtigsten EU-Rüstungsprojekten beteiligen und Battle Groups bereitstellen.

Schließlich wird mit dem Vertrag von Lissabon die Stimmverteilung im wichtigsten EU-Gremium, dem Rat der Staats- und Regierungschefs, massiv zugunsten der Großmächte verschoben. Von der neuen Regelung profitieren vor allem die bevölkerungsreichsten Staaten, allen voran Deutschland, das hierdurch seinen Stimmanteil von $8,4 \%$ auf $16,73 \%$ nahezu verdoppelt. Aber auch Frankreich, Großbritannien und Italien zählen zu den Gewinnern. Die Tragweite dieser Machtverschiebung nach Inkrafttreten des Lissabonner Vertrags ist kaum zu überschätzen: »Die Union [wird] danach eine andere werden. Die Mitgliedstaaten verlieren weiter an Souveränität, die großen Länder werden auf Kosten der kleinen gestärkt, und die Zentralisierung ihrer Entscheidungsstrukturen wird die EU noch undemokratischer machen. Sie droht, ihren Charakter als Aushandlungsgemeinschaft $\mathrm{zu}$ verlieren und eine feste Hegemonialordnung von Metropole und Peripherie zu werden. «Is Dies bedeutet nichts anderes als das altbekannte Kerneuropakonzept, nur diesmal konkret umgesetzt.

\section{Militärische Robstoffsicherung}

Aufgrund der schwindenden Weltölvorkommen bei gleichzeitig rapide wachsender Nachfrage, rückt die militärische Energiesicherung nicht nur in den USA, sondern auch innerhalb der Europäischen Union immer stärker in den Mittelpunkt der Strategieplanung. Im European Defence Paper, dem Entwurf für ein EU-Militär-Weißbuch, werden Rohstoffkriege bereits unverblümt ins Auge gefasst: »Künftige regionale Kriege könnten europäische Interessen tangieren [...], indem Sicherheit und Wohlstand direkt bedroht werden. Z.B. durch die Unterbrechung der Ölversorgung und/oder einer massiven Erhöhung der Energiekosten, [oder] der Störung der Handels- und Warenströme.«Konkret 
wird daraufhin folgendes Szenario beschrieben: »In einem Land x, das an den indischen Ozean grenzt, haben anti-westliche Kräfte die Macht erlangt und benutzen Öl als Waffe, vertreiben Westler und greifen westliche Interessen an.«Ziel sei es in diesem Fall, »das besetzte Gebiet zu befreien und die Kontrolle über einige der Ölinstallationen, Pipelines und Häfen des Landes x zu erhalten. «16

\section{Die Militärische Absicherung der Globalisierung}

Obwohl die kapitalistische Globalisierung und die sie begleitenden Politiken zu einer dramatischen Verarmung weiter Teile der Weltbevölkerung geführt hat, versucht die Europäische Union seit einigen Jahren immer aggressiver, ihre Liberalisierungsagenda im europäischen Großraum und, das stellt die außenpolitische Entsprechung der Lissabon-Strategie dar, auch darüber hinaus durchzusetzen. In diesem Zusammenhang ist es mehr als zynisch, wenn der Lissabonner Vertrag in Art. 2 I (2) angibt, die Europäische Union bekenne sich zu dem »vorrangigen Ziel, die Armut zu beseitigen«, nur um im nächsten Satz als zentrale Maßnahme hierfür Folgendes zu benennen: »die Integration aller Länder in die Weltwirtschaft zu fördern, unter anderem auch durch den schrittweisen $\mathrm{Ab}$ bau internationaler Handelshemmnisse.«

Hierbei kommt dem Militär die Aufgabe zu, die dem neoliberalen Weltwirtschaftsmodell inhärenten Hierarchie- und Ausbeutungsverhältnisse abzusichern und weltweit durchzusetzen. Selbst die Weltbank räumt inzwischen ein, dass Armut - nicht etwa religiöse, ethnische oder sonstige Faktoren, wie zumeist suggeriert wird - der bei weitem einflussreichste Faktor für die gewaltsame Eskalation von Konflikten in der Dritten Welt darstellt. ${ }^{17}$ Während also die Globalisierung einerseits den westlichen Großkonzernen neue Profitmöglichkeiten eröffnete, müssen auf der anderen Seite die permanent produzierten Konflikte »befriedet « werden, um die Stabilität der globalen Ordnung zu garantieren.

Darüber hinaus gilt es, die erzielten »Fortschritte « in diesem Bereich irreversibel zu gestalten, wie ein Beitrag in der Zeitschrift "griephan global security«, die gegenwärtig versucht, sich als Zentralorgan an der Schnittstelle zwischen Sicherheitspolitik und Wirtschaft zu etablieren, verdeutlicht: »Zur Zeit ist eines der größten Risiken, dass die gegenwärtig Ausgeschlossenen irgendwann Gehör finden und ihren Einfluss dann auf nationaler Ebene wieder geltend machen. Daraus entsteht sowohl für globale Unternehmen als auch für Investoren eine Herausforderung: Wie schützt man globale Unternehmensstrukturen in einer Zeit, wo sich das >Länderrisiko< im weitesten Sinne verschärft? Unternehmen müssen [sich] gegen politische und soziale Unruhen in den Nationalstaaten sichern. $\ll^{18}$

\section{Die Imperiale Ordnung des Robert Cooper}

Sollte diese wirtschaftliche Expansionsstrategie jedoch auf Widerstände stoßen, entweder in Form von sozialen Unruhen, Armutskonflikten, die sich in gewaltsamen Konflikten niederschlagen, oder renitenten Regierungen, die sich dem EU-

I6 André Dumoulin u.a.: European Defence - A Proposal for a White Paper, Report of an independent Task Force, Paris, Mai 2004, 8I ff.

I7 World Bank, Breaking the Conflict Trap: Civil War and Development Policy, Oxford 2003.

I 8 David Bowers, Nationale Bedrohungen für globale Bestrebungen, in: griephan global security, Herbst $2007,8-\mathrm{I} 4$, I0. 
Liberalisierungsprojekt widersetzen, ist die Union zunehmend bereit, ihr Militär zur Aufrechterhaltung der imperialen Ordnung einzusetzen. Dies legen etwa die Aussagen Robert Coopers, seines Zeichens Büroleiter Javier Solanas und Hauptautor der Europäischen Sicherheitsstrategie, nahe: »Der postmoderne Imperialismus hat zwei Komponenten. Die erste ist der freiwillige Imperialismus der globalen Ökonomie. Er wird normalerweise von einem internationalen Konsortium durch internationale Finanzinstitutionen wie IWF und Weltbank ausgeübt [...] Die zweite Dimension des postmodernen Imperialismus könnte der Imperialismus des Nachbarn genannt werden. Instabilität in der Nachbarschaft stellt eine Gefahr dar, die kein Staat ignorieren kann. Politische Misswirtschaft, ethnische Gewalt und Kriminalität auf dem Balkan stellen eine Gefahr für Europa dar. Die Antwort hierauf war, eine Art freiwilliges UN-Protektorat im Kosovo und in Bosnien zu schaffen. «19 Damit redet der EU-Spitzenpolitiker einer de facto ReKolonisierung der Peripherie das Wort, die keineswegs freiwillig erfolgt.

Darüber hinaus geht es Cooper nicht nur darum, die imperiale Ordnung im europäischen Großraum abzusichern, sondern sie auch gegenüber dem Rest der Welt durchzusetzen: »Die Herausforderung der postmodernen Welt ist es, mit der Idee doppelter Standards klarzukommen. Unter uns gehen wir auf der Basis von Gesetzen und offener kooperativer Sicherheit um. Aber wenn es um traditionellere Staaten außerhalb des postmodernen Kontinents Europa geht, müssen wir auf die raueren Methoden einer vergangenen Ära zurückgreifen - Gewalt, präventive Angriffe, Irreführung, was auch immer nötig ist, um mit denen klarzukommen, die immer noch im I9. Jahrhundert leben, in dem jeder Staat für sich selber stand. Unter uns halten wir uns an das Gesetz, aber wenn wir im Dschungel operieren, müssen wir ebenfalls das Gesetz des Dschungels anwenden. «०

Vor diesem Hintergrund häufen sich nassforsche Plädoyers wie etwa vom Chefkolumnisten der Welt am Sonntag, die Europäische Union müsse das »Imperium der Zukunft « werden und künftig z.B. als »imperiale Stabilisierungsmacht « in Afrika agieren. ${ }^{21}$ Auf eine perfide Weise ist dies nur konsequent. Denn solange keine Bereitschaft zur Veränderung der herrschenden Hierarchie- und Ausbeutungsverhältnisse besteht, wird wenig anderes übrig bleiben, als diese imperiale Ordnung militärisch gegen die »Verdammten dieser Erde ${ }^{22}$ abzusichern.

I9 Robert Cooper, The Post-Modern State, in: Mark Leonard (ed.), Re-Ordering the World, London 2002, I I-20, I 8 .

20 Ebd., I6.

2 I Alan Posener, Globale Politik - Warum Europa das Zeug zur Weltmacht hat, Die Welt, i6.9.2007.

22 Frantz Fanon, Die Verdammten dieser Erde, Frankfurt am Main 2009 (I96I). 